\title{
Characterization of Jets in Relativistic Heavy Ion Collisions
}

\author{
S. C. Phatak*and P. K. Sahu ${ }^{\dagger}$ \\ Institute of Physics, Bhubaneswar 751 005, India
}

\begin{abstract}
Jet quenching is considered to be one of the signatures of the formation of quark gluon plasma. In order to investigate the jet quenching, it is necessary to detect jets produced in relativistic heavy ion collisions, determine their properties and compare those with the jets one obtains in hadron-hadron or $e^{+}-e^{-}$collisions. In this work, we propose that calculation of flow parameters may be used to detect and characterize jets in relativistic heavy ion collisions.
\end{abstract}

PACS: 12.38.Mh, 24.85.+p, 25.75.-q

\section{Introduction}

The quenching of jets in relativistic heavy ion collisions has been proposed to be one of the signatures of the formation of quark gluon plasma (QGP) [1- [4. For a jet produced in heavy ion collision, the leading energetic parton of the jet will have to traverse through a medium consisting of QGP before it fragments and a jet is produced. During its passage through the plasma, it may loose some of its energy because of its interaction with the constituents of the plasma. It would also produce quarks and gluons during this period [1]-[5]. This effect seems to have been observed in the recent experiments [6, [7, although the observation is somewhat indirect. The quenching of the jet is expected to have several consequences on the characteristics of the jet. For example, one expects that the energy carried by the hadrons constituting the jet should decrease due to jet

*email: phatak@iopb.res.in

$\dagger$ †email: pradip@iopb.res.in 
quenching. This is because the leading parton of the jet looses its energy via gluon radiation while traversing through QGP and that results in the decrease in the energy carried by the hadrons in the jet. Another possible consequence of jet quenching is, the number of hadrons produced in the jet might increase because secondary quarks and gluons that are produced during the passage of the leading parton through the plasma. One more consequence of the interaction of the leading parton in the plasma is that there would be an increase in the opening angle of the jet. This would lead to a larger spread of the jet particles in azimuthal angle as well as rapidity.

The properties of jets produced in $e^{+}-e^{-}$and hadron-hadron collisions have been well investigated [8, 9] and the systematics of number of particles in a jet and the opening angle of the jet have been done. There is also a good theoretical understanding of these in terms of perturbative QCD and jet fragmentation functions [9, 10. In case of nucleus-nucleus collisions, on the other hand, the identification and characterization of jets are very difficult because during the collision process, a large number of hadrons are produced and the particles belonging to the jet constitute a very small fraction of these. Therefore, one cannot easily isolate the particles produced in a jet from the background particles and determine the properties of jets in a simple fashion. The jet characterization is made further difficult by small probability of jet production. Assuming a conservative value of $100 \mathrm{nb}$ as the hard jet production cross section [9] in nucleon-nucleon collision, the probability of jet production in central gold-gold collision is about $10^{-3}$. Indeed the particles produced in a jet have large momenta and they are emitted in a narrow cone in $\eta$ ( the rapidity ) and $\phi$ ( the azimuthal angle ) and the background particles are distributed over a large range of $\eta$ and spread more or less uniformly in $\phi$. But there is no obvious method of removing the background particles to extract the properties of the jet. Typically, the dispersion in the rapidity and azimuthal angle of the jet particles is quite small ( $\Delta=\sqrt{\Delta_{\eta}^{2}+\Delta_{\phi}^{2}}$ with $\Delta_{\eta} \sim \Delta_{\phi} \leq 0.5$ ). It would be useful to characterize the jets produced in heavy ion collisions in terms of the quantity $\Delta$ defined above as well as in terms of the number of particles in the jet and the energy carried by the jet particles. This will give a direct measure of jet quenching and jet fragmentation function. Further, measurement of these quantities as a function of impact parameter ( which is related to the total multiplicity or transverse energy in an event ) would also be of interest. This is because the amount of quark matter traversed by the leading parton in the jet, and therefore the amount of jet quenching is expected to depend on the impact parameter. Further, in case of large impact parameter collisions, one would expect that the quenching would be different if the jet direction is in or out of the reaction plane.

Methods, such as tagging the jet with photons [11] or di-leptons [11, 12] have been 
proposed to detect the jets and determine their properties in relativistic heavy ion collisions. These methods are indirect and they do not give full information on the jets. In this note, we have proposed a method of locating and identifying a jet from the data on hadron momentum distributions for a given event. The method uses the flow parameters [13] for this purpose. Our analysis indicates that the method is capable of estimating the number of particles in the jet as well as the jet opening angle reasonably well. We show that with the analysis of flow parameters for different transverse energy

cuts, it is possible to estimate the number of particles in a jet and the opening angle of the jet to some extent.

Investigation of flow parameters in heavy ion collisions is being done for quite some time 14. The main reason of this analysis is to determine the collective motion of the particles produced in the collisions. The focus here is on directed and elliptical flows which are expected to shed some light on the equation of state of the hot and dense matter. The flow introduced by jets is not exactly a collective effect. So, here we are advocating the measurement of flow for very different physical effect.

In the following, we describe the method in Section II, apply the method to synthetic data as well as simulated data 15 in Section III and describe the results and conclude in Section IV.

\section{The Method}

Given the transverse momentum distribution of particles produced in an event of relativistic heavy ion collision, one can compute the quantities $b_{m}\left(p_{0}\right)$ for all the particles having transverse momentum larger than some transverse momentum $p_{0}$;

$$
\begin{aligned}
b_{m}\left(p_{0}\right) & =\frac{\sum_{j} \cos (m \phi(j))}{\sum_{j}} \\
& =\frac{1}{N\left(p_{0}\right)} \sum_{j, p_{T}(j)>p_{0}} \cos (m \phi(j)) .
\end{aligned}
$$

Here $\phi(j)$ is the azimuthal angle of $j^{\text {th }}$ particle, the summation index $j$ runs over all particles having transverse momentum larger than $p_{0}$ and $N\left(p_{0}\right)$ is the number of particles having transverse momentum larger than $p_{0}$. The coefficients $b_{m}$ are related to the flow coefficients ( see later ) and for different values of $m$, these have specific meaning. For example, $b_{1}$ and $b_{2}$ are related to the directed and elliptic flow respectively. Generally, the flow coefficients for $m$ larger than 2 are not considered useful. Here, however, we shall be arguing that these would be needed to get information about jets. 
The coefficients $b_{m}$ 's are also small and difficult to determine for $m>2$ and, therefore not emphasized in the literature. However, recently it has been argued that, the fourth order flow coefficient is important in understanding of the physics of extreme energy loss and getting the information on dynamical evolution of the system[16]. The reasons for introducing momentum cut will be explained later.

Theoretically the flow coefficients $v_{m}$ 's are measured with respect to the reaction plane. Their definition is:

$$
v_{m}=\left\langle\cos \left(m\left(\phi_{i}-\phi_{E}\right)\right)\right\rangle
$$

where $\phi_{E}$ defines the reaction plane and $\langle\cdots\rangle$ implies averaging of the quantity $\cdots$ over all particles. Since, in an experiment, it is not possible to define the reaction plane, the flow coefficients are obtained by the pairwise correlation of all the particles. It is easy to show that the square of the flow coefficients, $v_{m}^{2}$ are given by

$$
\begin{aligned}
v_{m}^{2} & =\left\langle\cos \left(m\left(\phi_{i}-\phi_{j}\right)\right)\right\rangle_{i, j} \\
& =\frac{\sum_{i, j} \cos \left(m\left(\phi_{i}-\phi_{j}\right)\right)}{N^{2}\left(p_{0}\right)} .
\end{aligned}
$$

We shall now consider the flow coefficients $v_{m}\left(p_{0}\right)$ 's for further discussions. If the particles produced in the reaction are uniformly distributed in azimuthal angles, theoretically all $v_{m}\left(p_{0}\right)$ 's are zero. In case of the experimental data $v_{m}^{p_{0}}$ approaches zero as the number of particles goes to infinity. Thus, because of statistical fluctuations, the experimentally determined values of $v_{m}\left(p_{0}\right)$ 's are always nonzero. On the other hand, if all the particles have same azimuthal angle $\phi$, all $v_{m}$ 's will be unity.

It turns out that the computation of pairwise correlated quantities $v_{m}^{2}$ 's of eq(3) not only removes the uncertainty in the knowledge of the reaction plane but helps in reducing the statistical fluctuations also.

To begin with, let us consider that the particles are distributed according to an azimuthal probability distribution function $P(\phi)$. The flow coefficients are then defined as 


$$
\begin{aligned}
v_{m}^{2} & =<\cos \left(m\left(\phi_{1}-\phi_{2}\right)\right)> \\
& =\frac{\int d \phi_{1} d \phi_{2} \cos \left(m\left(\phi_{1}-\phi_{2}\right)\right) P\left(\phi_{1}\right) P\left(\phi_{2}\right)}{\int d \phi_{1} d \phi_{2} P\left(\phi_{1}\right) P\left(\phi_{2}\right)} \\
& =\frac{\int d \phi_{1} \int d \phi_{2}\left[\cos \left(m \phi_{1}\right) \cos \left(m \phi_{2}\right)+\sin \left(m \phi_{1}\right) \sin \left(m \phi_{2}\right)\right] P\left(\phi_{1}\right) P\left(\phi_{2}\right)}{\int d \phi_{1} P\left(\phi_{1}\right) \int d \phi_{2} P\left(\phi_{2}\right)} .
\end{aligned}
$$

\section{$2.1 \quad$ One Jet}

Let us now consider an event in which a jet with $N_{J}$ number of particles are produced. In addition, let us assume that the event consists of $N_{b}$ non-jet ( background ) particles and the transverse momentum and the azimuthal angle of these particles is known. Let us further assume that the background particles are distributed uniformly in the azimuthal angle $\phi$ and the azimuthal angles of the jet particles are uniformly distributed within the azimuthal angle $\phi_{0}-\frac{\Delta \phi}{2}$ and $\phi_{0}+\frac{\Delta \phi}{2}$. We shall also assume that the particles in a specified rapidity window are chosen for the analysis. For such a case, the probability distribution function would be

$$
P(\phi)=P_{1}(\phi)+P_{2}(\phi)
$$

where

$$
\begin{array}{rlrl}
P_{1}(\phi) & =\frac{N_{b}}{2 \pi N} & 0<\phi<2 \pi \\
P_{2}(\phi) & =\frac{N_{J}}{\Delta \phi N} & \phi_{0}-\frac{\Delta \phi}{2}<\phi<\phi_{0}+\frac{\Delta \phi}{2}, \\
& =0 & & \text { otherwise }
\end{array}
$$

and $N=N_{b}+N_{J}$. For the probability distribution given above, the flow coefficients $v_{m}^{2}$, as defined in eq(41) can be computed analytically. For this, one may choose the x-axis along $\phi_{0}$. Further, all the integrals in the numerator of eq(4) vanish for $P_{1}(\phi)$ and we get

$$
v_{m}^{2}\left(p_{0}\right)=\frac{N_{J}^{2}}{N^{2}}\left[\frac{\sin \left(\frac{m \Delta \phi}{2}\right)}{\left(\frac{m \Delta \phi}{2}\right)}\right]^{2}=\frac{N_{J}^{2}}{N^{2}}\left[j_{0}\left(\frac{m \Delta \phi}{2}\right)\right]^{2} .
$$

The sin integral terms in eq(4) vanished due to symmetry distribution function $P_{1}$. If the opening angle $\Delta \phi$ is small enough, the Bessel function $j_{0}$ can be expanded in power series. Keeping first two terms we get,

$$
v_{m}^{2} \sim \frac{N_{J}^{2}}{N^{2}}\left[1-\frac{(\Delta \phi)^{2} m^{2}}{12}+O\left((\Delta \phi)^{4}\right)\right] .
$$


The procedure for obtaining the number of jet particles and the jet opening angle from the data are now clear. After the computation of the flow parameters $v_{m}$ 's, one has to fit $v_{m}^{2}$ with a polynomial in $m^{2}$ as shown in the eq(7) above. Then the intercept on y-axis

gives $N_{j}$ ( note that $N$ is known from the data ) and the coefficient of $m^{2}$ term is related to $\Delta \phi$.

Although the approximate equation for the flow ( eq(17) has been derived for uniform distribution, it can be generalized for arbitrary probability distribution of jet particles, provided that the probability function $P_{2}(\phi)$ is sharply peaked at some angle $\phi_{0}$. In such a case, we can choose the $\mathrm{x}$ axis along $\phi_{0}$ ( as before ) and expand the cosine function in powers of $\left(\phi_{1}-\phi_{2}\right)$. Keeping up to quadratic terms in the expansion, we get

$$
\begin{aligned}
v_{m}^{2} & =\int d \phi_{1} d \phi_{2} \cos \left(m\left(\phi_{1}-\phi_{2}\right)\right) P_{2}\left(\phi_{1}\right) P_{2}\left(\phi_{2}\right) \\
& =\int d \phi_{1} d \phi_{2}\left[1-\frac{m^{2}\left(\phi_{1}-\phi_{2}\right)^{2}}{2}\right] P_{2}\left(\phi_{1}\right) P_{2}\left(\phi_{2}\right) \\
& =\frac{N_{J}^{2}}{N^{2}}\left[1-m^{2} \sigma^{2}\right]
\end{aligned}
$$

Here $\sigma=\sqrt{\left\langle\phi^{2}\right\rangle-\langle\phi\rangle^{2}}$ is the variance of the distribution $P_{2}(\phi)$. Note that the variance $\sigma$ is related to the spread of the jet particles in azimuthal angle. Particularly for uniform distribution defined in eq(5) above, $\sigma^{2}=\Delta \phi^{2} / 12$. Thus, for any sharply peaked probability distribution functions of jet particles, we can plot $v_{m}^{2}$ vs $m^{2}$ and from a linear fit to the curve one would get the number of jet particles and the opening angle of the jet.

From the expressions of the flow coefficients eq(6, 17) it is clear that $v_{m}^{2}$ would have sufficiently large and measurable if the number of jet particles constitute a sufficiently large fraction of the total number of particles $N$. Generally, this will not be the case. However, the jet particles are expected to have large transverse momentum and the background particles have exponentially falling transverse momentum distribution. One can therefore use transverse momentum cut to eliminate a large fraction of background particles and enhance the fraction of jet particles. This is expected to improve the determination of the jet parameters. We have performed the calculations with different transverse momentum cuts and these results will be discussed in next the section.

\subsection{Two Jets}

Let us now consider a case when there are two jets in the given rapidity window. We shall assume that the number of jet particles are $N_{J_{1}}$ and $N_{J_{2}}$ and the respective opening 
angles are $\Delta \phi_{1}$ and and $\Delta \phi_{2}$. Without loss of generality, we can choose the jet angle of the first jet to be zero and that of the second jet to be $\Phi$. Then the probability distribution function for this case is

$$
P(\phi)=P_{1}(\phi)+P_{2}(\phi)+P_{3}(\phi)
$$

with

$$
\begin{aligned}
P_{1}(\phi) & =\frac{N_{b}}{2 \pi N} & 0<\phi<2 \pi \\
P_{2}(\phi) & =\frac{N_{J_{1}}}{\Delta \phi_{1} N} & -\frac{\Delta \phi_{1}}{2}<\phi<\frac{\Delta \phi_{1}}{2}, \\
& =0 \quad & \text { otherwise } \\
P_{3}(\phi) & =\frac{N_{J_{2}}}{\Delta \phi_{2} N} & \Phi-\frac{\Delta \phi_{2}}{2}<\phi<\Phi+\frac{\Delta \phi_{2}}{2}, \\
& =0 & \text { otherwise. }
\end{aligned}
$$

As in the previous case, one can evaluate the flow coefficients analytically. As before, the background particles do not contribute. The result is,

$$
\begin{aligned}
v_{m-2 J}^{2}= & {\left[j_{0}\left(\frac{m \Delta \phi_{1}}{2}\right)\right]^{2} \frac{N_{J_{1}}^{2}}{N^{2}}+\left[j_{0}\left(\frac{m \Delta \phi_{2}}{2}\right)\right]^{2} \frac{N_{J_{2}}^{2}}{N^{2}} } \\
& +\left[j_{0}\left(\frac{m \Delta \phi_{1}}{2}\right) j_{0}\left(\frac{m \Delta \phi_{2}}{2}\right) \cos (m \Phi)\right]\left[\frac{2 N_{J_{1}} N_{J_{2}}}{N^{2}}\right]
\end{aligned}
$$

The meaning of the three terms in the equation above are obvious. The first two terms arise when both of the particles are from jet 1 and jet 2 respectively and the last term arises from the case when one particle is from jet 1 and the other is from jet 2 . As mentioned earlier, when one of the particles is from background, the contribution to the flow coefficient vanishes.

Consider a special case when the opening angels of jet one with $N_{J_{1}}$ particles and jet two with $N_{J_{2}}$ particles are same. Then eq(10) reduces to

$$
v_{m-2 J}^{2}=\left[j_{0}\left(\frac{m \Delta \phi}{2}\right)\right]^{2}\left[\cos (m \Phi)\left(\frac{2 N_{J_{1}} N_{J_{2}}}{N^{2}}\right)+\left(\frac{N_{J_{1}}^{2}+N_{J_{2}}^{2}}{N^{2}}\right)\right] .
$$

When $\Phi$, the angle between two jets is zero, the result in eq(11) reduces to the single jet result (eq(6) with the number of jet particles $N_{J_{1}}+N_{J_{2}}$. A case of particular interest is when $\Phi$ is equal to $\pi$. For that case, $\cos (m \Phi)$ is $(-1)^{m}$ and therefore the flow coefficients for odd values of $m$ are much smaller than those for even values. In fact, if the number of particles in each jet are equal, the flow coefficients for odd m would vanish theoretically. 
Since a pair of jets are produced from a hard collision of two partons from two nuclei, $\phi$ for the two jets will be close to $\pi[17$. However, if there is substantial jet quenching, the leading partons will loose some momentum to particles in quark-gluon plasma. In that case, the angle $\Phi$ will differ from $\pi$. Thus, in the case of two jets, the behavior of $v_{m}^{2}$ as a function of $m$ will be of interest.

In general, there are five parameters to be determined ( number of particles in two jets, two opening angles and the angle between two jets ). Since one can measure $v_{m}$ 's for $m$ going from 1 to 5 or 6 ( at the most ), we do not expect that these parameters can be determined to sufficient accuracy. But, probably the most important quantity in case of two jet events is the angle between two jets and that can probably be inferred.

\subsection{More Than Two Jets}

Our theoretical analysis can be extended to events having more than two jets. We do not do this for the following reasons.

1. Unlike two jet events, we do not expect a possible correlation in the angles between the jets. In case of two jet events, momentum conservation implies that the angle between two jets is close to $\pi$. As mentioned in the preceding subsection, this

produces definite pattern in $v_{m}^{2}$ 's. In case of events with three and more jets, no such pattern is expected.

2. In nucleus-nucleus collisions, more than two jets will be produced when

a) a single nucleon-nucleon collision produces more than two jets

b) or when two uncorrelated nucleon-nucleon collisions produced jets.

The probability of latter is very small since, as estimated in introduction, the chance of (hard) jet production itself is small. In case of the former, the multiple jet production probability would be related to $\alpha_{s}$, the strong coupling constant. The estimates indicate that the ratio of number of events with three or more jets to those with two jets is about 0.1 when the jets have large transverse momentum [18.

3. In order to control the number of "background" particles, it is better to do our analysis in a restricted rapidity window. The jet particles are expected to have a rapidity distribution in $\Delta \eta \sim 0.5$ and therefore the $\eta$-window for the analysis should be between 0.5 and 1.0. Because of this, it is likely that some of the jets in a multi-jet events will lie outside this window. This would further reduce the possibility of encountering more number of jets during the analysis. 
4. Further, we do not think that it would be possible to extract meaningful information about the jets in multi-jet events because the number of parameters in this case are large (jet angle and width for each jet and relative angles between jets) and the number of flow coefficients which can be measured reliably is restricted to five or six (at the most).

It is however pertinent to ask if the flow analysis gives some information regarding the multi-jet events. In particular, one would like to know whether one would be able to classify the events into no jets, one jet, two jet and multi-jet events after the flow analysis. We believe that this may be possible with some level of confidence. For example, the events without a jet, which would constitute the bulk of the events, would yield the flow coefficients similar to those obtained for uniform random distribution of particles

(in azimuthal angle). For single jet events, the flow coefficients $v_{m}^{2}$ 's will be larger and decreasing with $m$. For two jet events, these would be oscillating and if the jet angle is close to $\pi$, the coefficients for odd values of $m$ would be small. For events with more than two jets, we expect the particle distribution to be closer to the random distribution.

\section{The Calculations And Discussions}

The background particles in an event are constructed by taking the output from an event generator. For this purpose we have used the generator [15] which uses dynamical hadron string cascade model. For our analysis, we have considered $130 \mathrm{GeV}$ collision of gold nuclei. To these 'background' particles, the jet particles are added by hand. We choose different numbers of jet particles and assume that their transverse momenta are larger than $2 \mathrm{GeV}$. The direction of the jet as well as the azimuthal angle of the jet particles are chosen randomly. We, however assume that the probability of the azimuthal angle of the jet particles is constant over the opening angle $\Delta \phi$. In the following we shall consider the analysis when the event does not have a jet, there is only a jet ( no background ) and both jet and background particles are present.

\subsection{Events Without Jet}

Let us first consider the case where the event consists of only the background particles and no jet. Theoretically, the values of the flow coefficients are all zero as the background particles are uniformly distributed. However the measured coefficients differ from zero because of the fluctuations. Fig(II) shows the plot of the square of the flow coefficients 
for one of the events. The figure shows that the flow coefficients are non-zero and sometimes negative. This clearly indicates that the observed flow coefficients are indeed due to fluctuations. Further more, the values of the flow coefficients are smaller for lower transverse momentum cutoff. This is because the number of particles, in case of the lower cutoff are larger and therefore the fluctuations are smaller.

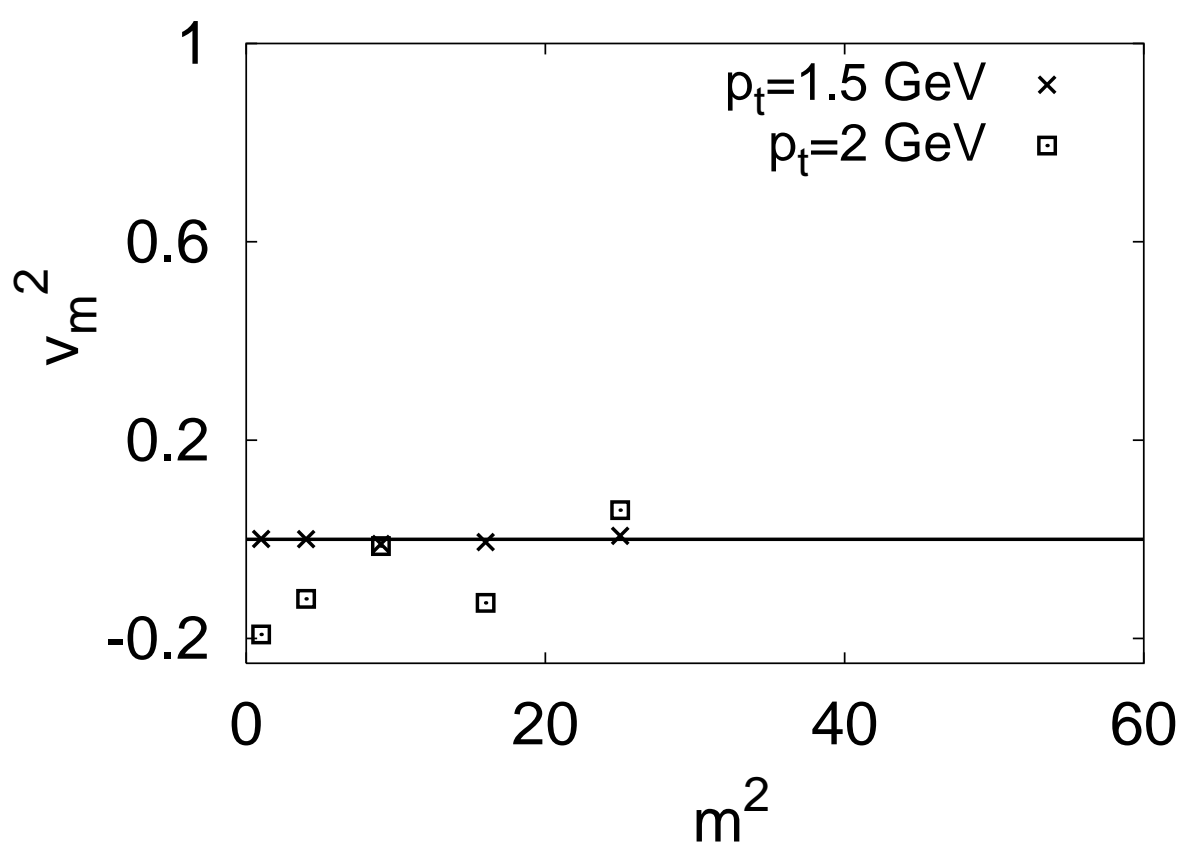

Figure 1: Plot of $v_{m}^{2}$ vs $m^{2}$ for the case of no jet. Note that the values of $v_{m}^{2}$ 's are close to zero for $P_{t}$ cut of $1.5 \mathrm{GeV}$. Also note that sometimes the extracted values of $v_{m}^{2}$ are negative, clearly indicating that these are essentially due to fluctuations.

The computed values of the flow coefficients for the events without a jet set a lower limit on the values of the flow coefficients from which meaningful information about jet characteristics can be determined. In particular, one may note that for smaller transverse momentum cutoff, the flow coefficients in absence of a jet are closer to zero as there are lot more background particles. So, in this case, in order to get reliable information, the flow coefficients in the presence of a jet need to be larger than the flow coefficients shown here. 


\subsection{Jet Only Events}

Let us now consider the case where there are no background particles. This, in a way, is an ideal case and we expect to obtain the best results from our analysis. Here we display the results for the extracted values of number of jet particles $\left(N_{o}\right)$ and the opening angle $\left(\Delta \phi_{o}\right)$ and compare these with the corresponding input values ( $N_{g}$ and $\Delta \phi_{g}$ respectively ) in Fig(2). The calculations are performed for different values of number of particles and opening angle of the jet. Also, the calculations are repeated a large number of times with different sets of background particles as well as jet particles so as to estimate the possible error in the extracted quantities. Fig(2) shows that the extracted values of the number of jet particles are very close to the input values. Also, the extracted values of the opening angle are close to the input values for $\Delta \phi$ smaller than 0.75 . Note that the expected opening angle of a jet is about this order. These results seem to indicate that it may be possible to determine the number of jet particles reasonably well. As for opening angles, we find that the determination may not be very much reliable if the opening angle exceeds 0.75 .
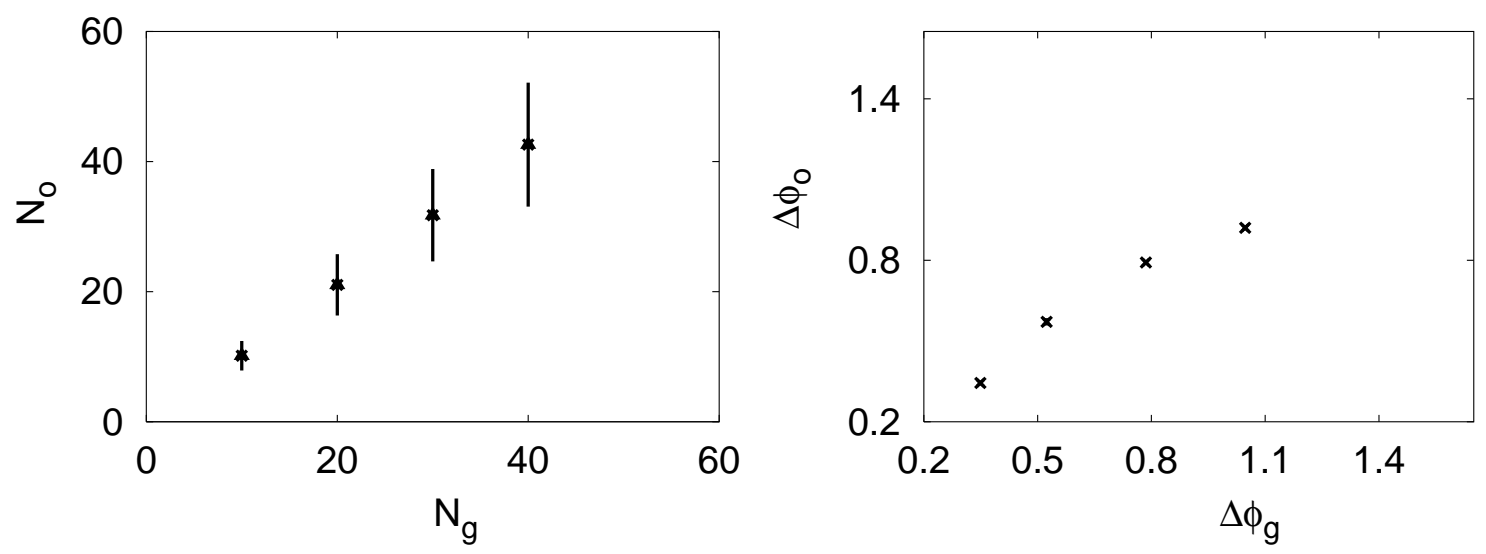

Figure 2: Plot of $v_{m}^{2}$ vs $m^{2}$ for the case of no jet.

\subsection{Events With Jet and Background}

We shall now consider the analysis of events in which a jet is produced in the presence of 'background' particles. For this analysis, a large number of events with impact parameters $1 \mathrm{fm}$ and $6 \mathrm{fm}$ ( corresponding to the central and peripheral events ) have been considered. The reason for choosing these two types of events is as follows. The number particles produced in a central event are large and this may make the determination of 
jet difficult. But such events are expected to have negligible elliptic flow. That is, the flow coefficients ( including the elliptic flow ) for the background particles are expected to be close to zero. This, on the other hand, is likely to help the jet characterization analysis. For the peripheral events, on the other hand, the number of background particles is smaller but the event are likely to have nonzero elliptic flow. The peripheral events are of interest for one more reason. For these events, the distance traveled by the leading parton in the jet is expected to be different for in-plane and out-of-plane direction of the parton. This would mean that the properties of jets in these two directions would be different if jet quenching is important.

It is clear that, for our method to work, the number of background particles should not be very large, so that the jet is not completely swamped by the background particles. One way of controlling the number of background particles is to remove the particles having transverse momenta smaller than certain value. This would remove background particles but not the jet particles if the cutoff momentum is sufficiently small. In fact, a very large fraction of the background particles have transverse momenta smaller than 1 $\mathrm{GeV}$. So, a transverse momentum cutoff larger than $1 \mathrm{GeV}$ would help in the analysis. On the other hand, the cutoff should not be too large since this may remove some of the jet particles also. Further more, when the number of background particles is very small, the fluctuations in the flow coefficients due to these background particles are large and this would affect the determination of the jet characteristics. We have therefore analyzed the events by including the background particles having transverse momenta larger than 1.5 and $2 \mathrm{GeV}$.
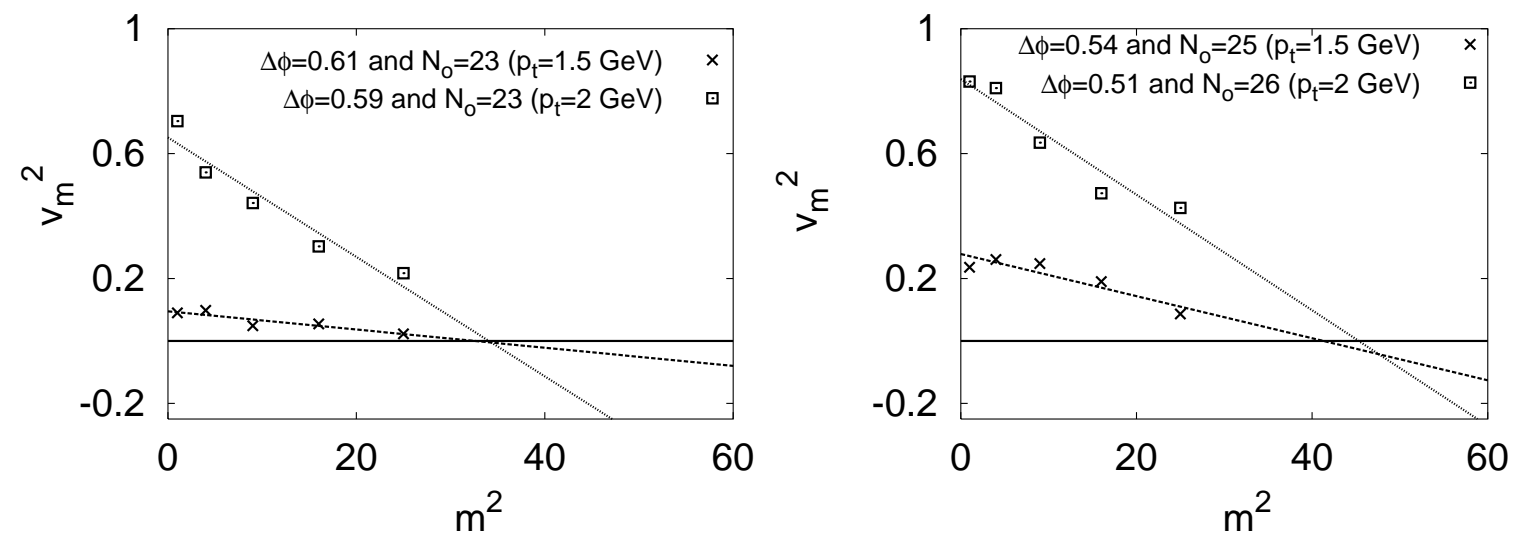

Figure 3: Plot of $v_{m}^{2}$ vs $m^{2}$. The left panel is for the central $\left(\mathrm{N}=74\right.$ and 28 for $p_{t}=1.5$ and $2 \mathrm{GeV}$, respectively) and the right panel is for the peripheral ( $\mathrm{N}=48$ and 28 for $p_{t}=1.5$ and $2 \mathrm{GeV}$, respectively) collisions. 
Fig(3) displays a plot of the square of the flow coefficients $v_{m}$ vs $m^{2}$ for the two transverse momentum cuts. The opening angle for this graph is $\pi / 6$ and the number of jet particles is 20 . The left panel is for the central collision and the right panel is for the peripheral collision. Note that the computed flow coefficients are significantly larger than those for no jet ( see Fig(1). Thus we expect that the extracted values of jet parameters are reliably determined. The extracted values of the number of jet particles and the opening angle are shown in the figure. We find that the extracted values of number of jet particles as well as the opening angle are within $25 \%$ of the input values.

The plots in Fig(3) are for the analysis of one particular event. We have repeated the analysis for a large number of such events. As expected, the extracted values fluctuate and from the fluctuations we have determined the errors in the extracted quantities. These are displayed in Fig(4).

The plot of the number of jet particles extracted from the analysis vs the corresponding input value is shown in the left panels of Fig(4). The top panel is for central collisions and the bottom panel is for peripheral collisions. The results are for the opening angle of $\pi / 6$. The plot shows that there is a linear correlation between the particle numbers extracted and the corresponding input numbers when the number of jet particles are larger than 10. Also, the correlation is better for $2 \mathrm{GeV}$ cutoff. We find that the results for central and peripheral collisions are similar.

The right panels of Fig(44) displays the extracted values of the opening angle vs the input opening angle. The results are for twenty jet particles. This figure shows that there is a correlation between extracted and input opening angles but it is not as strong as that for the number of jet particles. Particularly, the extracted opening angle does not increase linearly for larger input opening angles. One reason for this is that for larger angles, the expansion in the powers of $m^{2}$ fails for large $m$ and these results in the saturation of the computed opening angle.

The extracted values of the number of jet particles for different input opening angles have similar behavior as that observed in left panels of Fig(44). Thus, it seems that the extraction of the number of jet particles is quite robust but that of the jet opening angle from the flow analysis is less reliable.

We would like to note here that, although the extracted flow coefficients are smaller for $1.5 \mathrm{GeV}$ cutoff, one is still able to obtain some information about jets. This is because the extracted flow coefficients are still sufficiently larger than those for the background. When the cutoff is reduced below $1 \mathrm{GeV}$, we find that the extracted coefficients are extremely small. That is, the background particles essentially swamp the jet particles. In such cases a meaningful extraction of jet properties is not possible. We find that, in order 

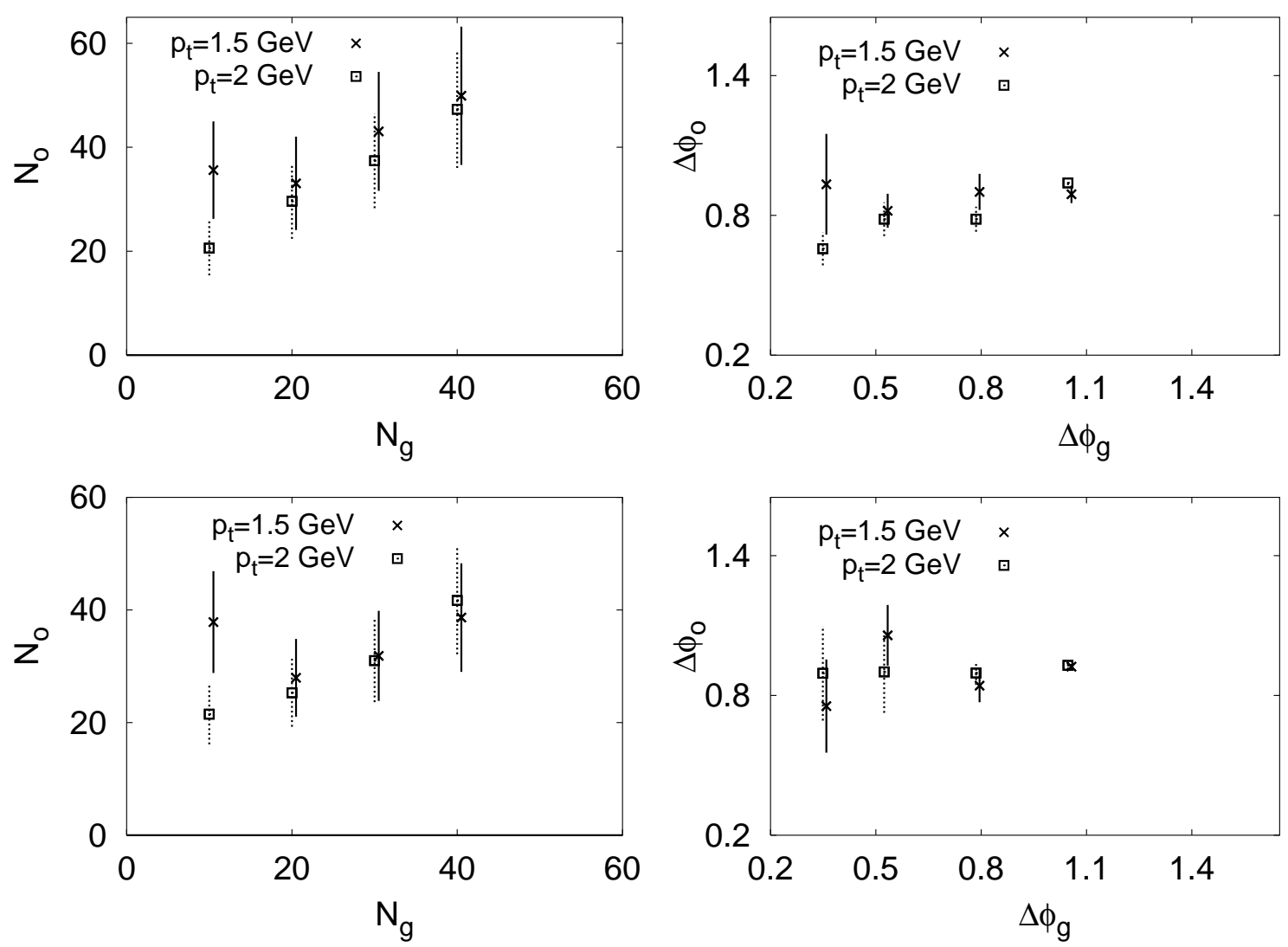

Figure 4: Plot of the extracted numbers of jet particles (opening angles) vs the corresponding input values are shown in left(right) panels. The top (bottom) panels are for the central (peripheral) collisions. The data points and error bars for $p_{t}=1.5 \mathrm{GeV}$ have been shifted marginally to distinguish from $p_{t}=2 \mathrm{GeV}$.

to obtain significant jet information, the number of jet particles should constitute more than $5 \%$ of the total number of particles. It should be apparent that this requirement reduces when the jet angle is smaller.

\subsection{Two Jets plus Background}

The computed flow coeficients when two jets are present are displayed in Table I(columns 6 through 9). The first three columns give transverse momentum cut, angle between the two jets $(\phi)$ and opening angles of the jets. The next two columns give the number of particles in the two jets. The angle $\phi$ between the two jets has ben chosen to be larger than $120^{\circ}$. As discussed in the previous section, we expect that the angle between two 
Table 1: particles from two jets, $v_{m-2 J}^{2}$ vs $m$ for different parameters.

\begin{tabular}{ccccccccc}
\hline$p_{t}(\mathrm{GeV})$ & $\phi$ & $\Delta \phi$ & $N_{J 1}$ & $N_{J 2}$ & $v_{1-2 J}^{2}$ & $v_{2-2 J}^{2}$ & $v_{3-2 J}^{2}$ & $v_{4-2 J}^{2}$ \\
\hline 1.5 & $\pi$ & $\frac{\pi}{6}$ & 10 & 20 & 0.00047 & 0.14 & 0.0091 & 0.196 \\
1.5 & $\pi$ & $\frac{\pi}{6}$ & 20 & 20 & 0.0069 & 0.18 & 0.00092 & 0.236 \\
2.0 & $\pi$ & $\frac{\pi}{6}$ & 10 & 20 & 0.096 & 0.74 & 0.123 & 0.597 \\
2.0 & $\pi$ & $\frac{\pi}{6}$ & 20 & 20 & 0.0008 & 0.78 & 0.0072 & 0.633 \\
\hline 2.0 & $\frac{2 \pi}{3}$ & $\frac{\pi}{6}$ & 10 & 20 & 0.265 & 0.206 & 0.519 & 0.198 \\
2.0 & $\frac{2 \pi}{3}$ & $\frac{\pi}{6}$ & 20 & 20 & 0.192 & 0.195 & 0.586 & 0.139 \\
2.0 & $\frac{5 \pi}{6}$ & $\frac{\pi}{6}$ & 10 & 20 & 0.146 & 0.543 & 0.410 & 0.163 \\
2.0 & $\frac{5 \pi}{6}$ & $\frac{\pi}{6}$ & 20 & 20 & 0.052 & 0.587 & 0.326 & 0.178 \\
\hline 2.0 & $\pi$ & $\frac{\pi}{9}$ & 10 & 20 & 0.097 & 0.779 & 0.132 & 0.746 \\
2.0 & $\pi$ & $\frac{\pi}{9}$ & 20 & 20 & 0.0007 & 0.819 & 0.0063 & 0.776 \\
jets only & $\pi$ & $\frac{\pi}{6}$ & 10 & 20 & 0.157 & 0.915 & 0.135 & 0.696 \\
jets only & $\pi$ & $\frac{\pi}{6}$ & 20 & 20 & 0.0025 & 0.921 & 0.0033 & 0.715 \\
\hline
\end{tabular}

jets is close to $\pi$. The opening angles of both the jets have been chosen to be equal.

We find that the determination of individual jet parameters from the flow analysis is not possible because the number of measured flow coefficients $v_{m}$ 's does not exceed 6 . Therefore a detailed analysis, like in the case of a single jet, cannot be done. Never the less, the table clearly shows that the flow coeficients for odd $m$ 's are generally smaller than those of even $m$ 's, particularly when the angle between the jets is $\pi$. It may be noted that this odd-even effect is noticable even when the number of particles in each jet differs significantly. But the effect reduces considerably when the angle is reduced to $150^{\circ}$ or less [19]. So, it seems that a good estimate of the angle between the two jets from the flow analysis may possible.

\section{Summary}

A method of extracting jet characteristics from flow analysis is discussed in this work. We have shown that when the data has a jet with the number of jet particles in excess of 10, the higher flow coefficients are large enough to be measured. In particular, we show that transverse momentum cuts can be used to enhance the effect of jet particles on the 
flow coeficients. Further, by analyzing the flow coefficients of different orders, we show that it is possible to extract the information of jet characteristics, namely the number of particles in the jet and jet opening angle. We believe that this is an interesting result and can be used to analyze the heavy ion collision data and extract the jet information. Our calculations show that in order to extract meaningful results, it is necessary to use transverse momentum cut to reduce the background. We show that a cut between 1.5 and $2 \mathrm{GeV}$ works well. This is nice since the jet particles are expected to have transverse momenta larger than these values.

Although we do not demonstrate, we believe that instead of the transverse momentum cuts, one may compute the transverse momentum ( or transverse energy ) weighted flow coefficients

$$
\begin{aligned}
b_{m}^{p_{T}}\left(p_{0}\right) & =\frac{\sum_{n} p_{T}(n) \cos (m \phi(n))}{\sum_{n}} \\
& =\frac{1}{N\left(p_{0}\right)} \sum_{n, p_{T}(j)>p_{0}} p_{T}(n) \cos (m \phi(j)) .
\end{aligned}
$$

One may even use higher powers of the transverse momentum in eqn(12) above. Actually, such flow coefficients do de-emphasize the particles having smaller momenta, which are predominantly the background particles. A bonus, in such calculations is that such flow coefficients will give information on the transverse momentum or energy carried by the jet particles.

We have also considered the case when two jets are present in the given rapidity window. For such a case, we have argued that an important information to be determined in this case is the angle between the two jets. We have shown that it may be possible to estimate this angle between the two jets.

The method developed in the present work is not useful to obtain the information of more than three jets. This is because the number of parameters in such a case are larger than the number of flow coefficients which can be measured meaningfully. However, the multi-jet events are expected to yield the flow coefficients which are similar to those obtained for the background particles.

In the present work, the jet particles have been introduced by hand. This has been done because, at this stage, we want to analyse jet events with known jet properties (number of particles and opening angles) so that we can determine how faithfully these are extracted from the analysis. The results are encouraging when one has a single jet. The computations also indicate that two-jet events are also identified if the angle between two jets is close to $\pi$. The next step is to use our procedure on the data created 
using generators which include jet production algorithm[20]. If this succeeds, one can think of applying the procedure to RHIC data.

\section{Acknowledgements}

One of us (PKS) would like to thank A. Ohnishi and Y. Nara for helpful discussions.

\section{References}

[1] X. N. Wang and M. Gyulassy, Phys. Rev. Lett. 68, 1480 (1992).

[2] R. Baier, Y. L. Dokshitzer, A. H. Mueller, S. Peigne and D. Schiff, Nucl. Phys. B 483, 291 (1997).

[3] B. Muller, Phys. Rev. C 67, 061901 (2003).

[4] M. Gyulassy, Nucl. Phys. B 571, 197 (2000).

[5] M. Gyulassy, Nucl. Phys. A 661, 637 (1999).

[6] K. Adcox, et al., [PHENIX Collaboration], Phys. Rev. Lett. 87, 052301 (2001).

[7] K. Adcox, et al., [PHENIX Collaboration], Phys. Rev. Lett. 88, 192303 (2002).

[8] B. Potter, Nucl. Phys. B 540, 382 (1999).

[9] R. K. Ellis, W. J. Stirling and B. R. Webber, QCD and Collider Physics, Cambridge University Press (1996).

[10] X. Wang, Nucl. Phys. A 702, 238 (2002).

[11] K. Gallmeister, B. Kamper and O.P. Pavlenko, Nucl. Phys. A 715, 705 (2003).

[12] D. K. Srivastava, C. Gale and T. C. Awes, Phys. Rev. C 67, 054904 (2003).

[13] N. Borghini, P. M. Dinh and J. -Y. Ollitrault, Phys. Rev. C 64, 054901 (2001).

[14] P. K. Sahu and W. Cassing, Nucl. Phys. A712, 357 (2002); P. K. Sahu, W. Cassing, U. Mosel, and A. Ohnishi, Nucl. Phys. A672, 376 (2000). 
[15] Y. Nara, N. Otuka, A. Ohnishi, K. Niita, and S. Chiba, Phys. Rev. C 61, 024901 (2000).

[16] P. F. Kolb, nucl-th/0306081.

[17] For the jets produced in $e^{+}-e^{-}$collisions, the angle between the two jets is $\pi$. In case of the jets produced in nucleus-nucleus collisions, the angle will be slightly different from $\pi$ as the two partons will have some transverse momentum before the hard collision. However, the deviation is expected to be small as the parton transverse momentum before the collision is not very large.

[18] F. Abe, et al., [CDF Collaboration], Phys. Rev. Lett. 75, 608 (1995).

[19] It is interesting to note that when $\phi$ the angle between two jets is $120^{\circ}, \cos 3 \phi$ is +1 and $v_{3}$ is largest.

[20] S. C. Phatak and P. K. Sahu, work in progress. 